PROCEEDINGS OF THE

AMERICAN MATHEMATICAL SOCIETY

Volume 34, Number 1, July 1972

\title{
MAXIMAL OPERATORS AND CAPACITY
}

\author{
DAVID R. ADAMS ${ }^{1}$
}

\begin{abstract}
It is shown that many maximal functions defined on the $L_{p}$ spaces are bounded operators on $L_{p}$ if and only if they satisfy a capacitary weak type inequality.
\end{abstract}

1. Throughout $X$ will denote either the $n$-dimensional torus or Euclidean $\boldsymbol{R}^{n}$. For each $\varepsilon>0$ and $r \in I, I$ some index set, let $\theta_{r}^{\varepsilon}(x)$ be a complex valued function of $x \in X$ belonging to $L_{q}(X)$, for all $q, 1<q<\infty$. Furthermore, assume that for each fixed $r \in I, \theta_{r}^{\varepsilon} * f \rightarrow F_{r}$ strongly in $L_{p}(X)$ as $\varepsilon \rightarrow 0, f \in L_{p}(X), 1<p<\infty$. The symbol $*$ denotes the usual convolution integration over $X$. Thinking of $\theta_{r}^{\varepsilon}$ as an approximation to a singular integral (convolution) operator $\theta_{r}$ on $L_{p}(X)$ for each $r \in I$, we write $F_{r}=\theta_{r} * f$. If $\theta_{r} \in L_{q}$, for all $q, 1<q<\infty$, we permit $I$ to be arbitrary, otherwise assume $I$ is countable. Define the maximal operator $M(f)(x)$ by:

$$
M(f)(x)=\sup _{r \in I}\left|\theta_{r} * f(x)\right|
$$

and assume $M(f)$ is Lebesgue measurable on $X$. The theory for $\theta_{r}^{e}, \theta_{r}$, and $M$ given here is modeled after the various maximal operators occurring in the literature, in particular when $\theta_{r} \in L_{q}$, for all $q, 1<q<\infty, \theta_{r}$ could be an "average" (as with the Hardy-Littlewood maximal function on $\boldsymbol{R}^{1}$ ) or a Dirichlet kernel (as with regard to "partial sums" of multiple Fourier series-see [4], [6], [9]). When $\theta_{r}$ is singular, interesting examples are

$$
\theta_{n}(x)=x^{-1} e^{-i n x}, \quad x \in X, n=\text { integer, }
$$

or the various "modified Dirichlet" kernels appearing in [9]. In general such operators satisfy a maximal inequality:

$$
\|M(f)\|_{p, X} \leqq C_{p}\|f\|_{p, X}
$$

Received by the editors July $18,1971$.

AMS 1970 subject classifications. Primary 31B15, 42A20, 42A92; Secondary 43A15, 44A25.

Key words and phrases. Capacity, maximal functions, Fourier series, singular integrals, $L_{p}$ spaces.

1 This research was partially supported by NSF GP 23400.

(c) American Mathematical Society 1972 
for all $f \in L_{p}(X), 1<p<\infty, C_{p}$ independent of $f$. The norms are $L_{p}(X)$ norms.

The main result states that inequalities of type (2) are equivalent to a capacitary weak type inequality. This is Theorem 1 of $\$ 2$. In $\$ 3$ some remarks concerning the results appearing in [3] and [8] are made, in particular it is possible to view these as corresponding to our case $p=2$.

2. By a capacity $C$ we will mean a nonnegative extended real valued set function defined on a $\sigma$-additive class of sets of $X$ which contains the compact sets and such that (i) $C(\varnothing)=0, \varnothing=$ empty set, (ii) $C(A) \leqq C(B)$, for $A \subset B$, (iii) $C\left(\bigcup_{i} A_{i}\right) \leqq \sum_{i} C\left(A_{i}\right)$. In particular, we will be interested in:

Definition. For $k \in L_{\mathbf{1}}^{+}\left(\boldsymbol{R}^{n}\right)$ and lower semicontinuous, let

$$
C_{k, p}(A)=\inf \|f\|_{p, X}^{p}, \quad 1<p<\infty,
$$

where the infimum is over all $f \in L_{p}^{+}(X)$ such that $k * f(x) \geqq 1$ on $A, A \subset X$.

The set functions $C_{k, p}$ are capacities and have been studied extensively in [7]. A property of $C_{k, p}$ proved in [7] and used below is: if $f_{k} \rightarrow f$ strongly in $L_{p}, k=1,2, \cdots$, then there is a subsequence $f_{k}^{\prime}$ such that $k * f_{k}^{\prime} \rightarrow$ $k * f, C_{k, p}$-a.e.

THEOREM 1. Suppose $M$ is the maximal operator defined in $\S 1$ and satisfies (2), $1<p<\infty$. Then

$$
C_{k, p}[M(k * f)>t] \leqq C_{p}^{p} t^{-p}\|f\|_{p, X}^{p}, \quad t>0,
$$

for all $f \in L_{p}(X), 1<p<\infty$. Conversely, if (3) holds, then (2) holds for some constant $C_{p}^{\prime}$ independent of $f, 1<p<\infty$.

We say that $M(k * \cdot)$ is of capacitary weak type $(p, p)$ when (3) holds. An important feature in (3) is that $C_{p}$ is independent of both $f$ and $k$. This allows us to view the usual weak type inequality

$$
m[M(f)>t] \leqq C_{p}^{p} t^{-p}\|f\|_{p, X}^{p},
$$

$m=$ Lebesgue measure, as a limiting case of (3) in light of

THEOREM 2. Let $k_{i} \geqq 0, i=1,2, \cdots$, and satisfy

(a) $\int_{R^{n}} k_{i}(t) d t \rightarrow 1$, as $i \rightarrow \infty$,

(b) $\int_{|t| \geqq \eta} k_{i}(t) d t \rightarrow 0$, as $i \rightarrow \infty$, for all $\eta>0$, then $C_{k_{i}, p}(K) \rightarrow m(K), K$ compact in $X$.

Proof of Theorem 1. If $\theta_{r} \in L_{q}, 1<q<\infty$, then

$$
\theta_{r} *(k * f)(x)=k *\left(\theta_{r} * f\right)(x)
$$

for all $x \in X$. Otherwise, if $\theta_{r}^{\varepsilon} * f \rightarrow \theta_{r} * f$ in $L_{p}$, then there is a sequence 
$\varepsilon_{j} \rightarrow 0$ as $j \rightarrow \infty$ such that

$$
\theta_{r}^{\varepsilon_{j}} *(k * f)(x)=k *\left(\theta_{r}^{\varepsilon_{i}} * f\right)(x) \rightarrow k *\left(\theta_{r} * f\right)(x),
$$

$C_{k, p}$-a.e. Hence $\theta_{r} *(k * f)(x)=k *\left(\theta_{r} * f\right)(x), C_{k, p}$-a.e. on $X$. Of course the exceptional set depends on $r$. Call it $E_{r}$. Then

$$
\left|\theta_{r} *(k * f)(x)\right| \leqq k *\left|\theta_{r} * f\right|(x), \quad x \notin E_{r},
$$

and $C_{k, p}\left(E_{r}\right)=0$. Hence

$$
M(k * f)(x) \leqq k * M(f)(x), \quad C_{k, p} \text {-a.e. }
$$

since $C_{k, p}\left(\bigcup_{r \in I} E_{r}\right) \leqq \sum_{r \in I} C_{k, p}\left(E_{r}\right)=0$. It now follows easily from the definition of $C_{k, p}$ that

$$
C_{k, p}[M(k * f)>t] \leqq C_{k, p}[k * M(f)>t] \leqq t^{-p}\|M(f)\|_{p, X}^{p} \leqq C_{p}^{p} t^{-p}\|f\|_{p, X}^{p} .
$$

For the converse, first note that if $k * f \geqq 1$ on $A$,

$$
m(A) \leqq \int_{A} k * f d x \leqq\|k * f\|_{p, X} \cdot m(A)^{1 / p^{\prime}} \leqq\|k\|_{1}\|f\|_{p, X} m(A)^{1 / p^{\prime}}
$$

Thus if $m(A)<\infty, m(A) \leqq\|k\|_{1}^{p} C_{k, p}(A)$. This inequality also holds when $m(A)=\infty$ since $C_{k, p}(A)=\infty$ as a result. We now choose $k=g_{\alpha}$, where $g_{\alpha}, \alpha>0$, is the $L_{1}^{+}$function on $R^{n}$ which is the Fourier transform of $(2 \pi)^{-n / 2}\left(1+|\xi|^{2}\right)^{-\alpha / 2}, \xi \in \boldsymbol{R}^{n}$. Clearly $\left\|g_{\alpha}\right\|_{1}=1$. Thus

$$
m\left[M\left(g_{\alpha} * f\right)>t\right] \leqq C_{g_{\alpha}, p}\left[M\left(g_{\alpha} * f\right)>t\right] \leqq C_{p}^{p} t^{-p}\|f\|_{p, X}^{p}
$$

Note, $g_{\alpha} * f(x)-g_{\beta} * f(x)=g_{\sigma} *\left[g_{\alpha-\sigma} * f-g_{\beta-\sigma} * f\right](x), g_{0} * f=f, \alpha, \beta>0, \quad \sigma=$ $\min (\alpha, \beta)$. Thus

$$
m\left[M\left(g_{\alpha} * f-g_{\beta} * f\right)>t\right] \leqq C_{p}^{p} t^{-p}\left\|g_{\alpha-\sigma} * f-g_{\beta-\sigma} * f\right\|_{p}^{p} .
$$

Since $g_{\delta} * f \rightarrow f$ strongly in $L_{p}$ as $\delta \rightarrow 0^{+}$( $g_{\delta}$ is an approximation of the identity-see [2] for the necessary properties of $g_{\delta}$ ), we have, by the sublinearity of $M, M\left(g_{\alpha} * f\right)$ is Cauchy in measure as $\alpha \rightarrow 0$. Thus $M\left(g_{\alpha} * f\right) \rightarrow h$ in measure as $\alpha \rightarrow 0$ and $m[h>t] \leqq C_{p}^{p} t^{-p}\|f\|_{p, X}^{p}$. But $\theta_{r} *\left(g_{\alpha} * f\right)(x) \leqq$ $M\left(g_{\alpha} * f\right)(x)$, all $x$, and $\theta_{r} *\left(g_{\alpha} * f\right)(x) \rightarrow \theta_{r} * f(x)$, all $x$, when $\theta_{r} \in L_{q}, 1<q<\infty$, or only a.e. for some sequence $\alpha_{j} \rightarrow 0$, when $\theta_{r}$ is singular. Hence

$$
\theta_{r} * f(x) \leqq h(x), \quad x \notin E_{r}, m\left(E_{r}\right)=0 .
$$

But then $M(f)(x) \leqq h(x)$, a.e. Thus $M(\cdot)$ is of usual weak type $(p, p)$, $1<p<\infty$, and the desired result follows by the Marcinkiewicz interpolation theorem. Q.E.D. 
CoRollary. $\int M(k * f) d \mu \leqq C_{p}\|f\|_{p, X} \cdot I_{k, p}(\mu)^{1 / p^{\prime}}$ is equivalent to (2), $1<p<\infty$, where $I_{k, p}(\mu)$ is the generalized energy of $\mu, \mu$ a positive Borel measure, i.e.,

See [7].

$$
I_{k, p}(\mu)=\int k *(k * \mu)^{1 /(p-1)} d \mu
$$

Proof of Theorem 2. We have already noted $m(K) \leqq\|k\|_{1}^{p} C_{k, p}(K)$, hence $m(K) \leqq \lim \inf _{i \rightarrow \infty} C_{k_{i}, p}(K)$.

For the other inequality, note that for the given $K$ we can take a bounded open set $G \supset K$ and from (b): for each $\varepsilon, 0<\varepsilon<1$, there is an $i_{0}$ such that $k_{i} * \chi_{G}(x) \geqq 1-\varepsilon$ on $K$, when $i \geqq i_{0}, \chi_{G}=$ characteristic function of $G$. Thus

$$
\begin{aligned}
C_{k_{i}, p}(K) & \leqq m(G) /(1-\varepsilon)^{p}, \\
\therefore \quad \limsup _{i \rightarrow \infty} C_{k_{i}, p}(K) & \leqq m(G) /(1-\varepsilon)^{p},
\end{aligned}
$$

and by the arbitrariness of $G$ and $\varepsilon$ the result follows.

REMARK. If $\theta_{r} * f \rightarrow f$, a.e., for $f$ in a dense class of $L_{p}$ and (3) holds, then $\theta_{r} *(k * f) \rightarrow k * f, C_{k, p}$-a.e. for all $f \in L_{p}, 1<p<\infty$.

3. For $k \in L_{1}^{+}\left(\boldsymbol{R}^{n}\right)$, define

$$
c_{k, p}(K)=\sup \|\mu\|_{1}, \quad 1 \leqq p<\infty,
$$

where the supremum is over all nonnegative Radon measures $\mu\left(\mu \in \mathscr{M}^{+}\right)$ for which supp $\mu \subset K, K$ compact, and $\|k * \mu\|_{p^{\prime}, X} \leqq 1$. Here $\|\mu\|_{1}$ is the total variation of $\mu$. The capacity $c_{k, p}$ is also studied in [7] where, for all analytic sets $A, c_{k, p}(A)=C_{k, p}(A)^{1 / p}$ is established, $1<p<\infty$. For $p=1$, $C_{k, 1}$ is defined using $\mathscr{L}_{1}^{+}$in place of $L_{p}^{+}$, i.e., $\mu \in \mathscr{M}^{+}$with $\|\mu\|_{1}<\infty$. For compact $K, C_{k, 1}(K)=c_{k, 1}(K)$. See [5]. Thus when $p=2$ and when it is possible to write $k=k_{0} * k_{0}, k_{0} \in L_{1}^{+}, k_{0}(x)=k_{0}(|x|)$ and decreasing as $|x| \uparrow \infty$, then

$$
C_{k, 1}(A) \sim C_{k_{0}, 2}(A), \quad \text { all } A \subset X .
$$

Here the symbol $\sim$ means the ratio is bounded above and below by positive finite constants independent of $A$. (Use the "boundedness principle" of [1] to prove this.)

Also, if $\mathfrak{F}$ denotes the Fourier transform in $\boldsymbol{R}^{n}$, and $H_{\boldsymbol{k}}$ consists of all $u \in L_{2}(X)$ such that

$$
\|u\|_{k}=\left(\sum_{-\infty}^{\infty}|\hat{u}(n)|^{2}|\mathfrak{F} k(n)|^{2}\right)^{1 / 2}<\infty,
$$

^ denoting Fourier coefficients, then each $u \in H_{k}$ has an $L_{2}$ representative 
as $k * f, f \in L_{2}(X)$, and $\|u\|_{k}=\|f\|_{2, X}$. With these facts, it is possible to view the results of [3] and [8] as included in the case $p=2$ of Theorem 1.

Finally, we note that if $k=g_{\alpha}$, the classical theorem of Beurling-SalemZygmund becomes

$$
S_{N}\left(g_{\alpha} * f\right)(x) \rightarrow g_{\alpha} * f(x), \quad C_{g_{\alpha}, 2} \text {-a.e. }
$$

$S_{N}$ the $N$ th partial sum of the Fourier series of $g_{\alpha} * f, f \in L_{2}(-\pi, \pi)$. See [10, p. 195].

\section{REFERENCES}

1. D. R. Adams and N. G. Meyers, Bessel potentials. Inclusion relations among classes of exceptional sets, Bull Amer. Math. Soc. 77 (1971), 968-970.

2. A.-P. Calderón, Lebesgue spaces of differentiable functions and distributions, Proc. Sympos. Pure Math., vol. 4, Amer. Math. Soc., Providence, R.I., 1961, pp. 33-49. MR 26 \#603.

3. L. Carleson, Maximal functions and capacities, Ann. Inst. Fourier (Grenoble) 15 (1965), fasc. 1, 59-64. MR 32 \#2602.

4. C. Fefferman, On the convergence of multiple Fourier series, Bull. Amer. Math. Soc. 77 (1971), 744-745.

5. B. Fuglede, Le théorème du minimax et la théorie fine du potentiel, Ann. Inst. Fourier (Grenoble) 15 (1965), fasc. 1, 65-88. MR 32 \#7781.

6. R. A. Hunt, On the convergence of Fourier series, Proc. Conference Orthogonal Expansions and their Continuous Analogues (Edwardsville, Ill., 1967), Southern Illinois Univ. Press, Carbondale, Ill., 1968, pp. 235-255. MR 38 \#6296.

7. N. G. Meyers, $A$ theory of capacities for potentials of functions in Lebesgue classes, Math. Scand. 26 (1970), 255-292.

8. C. Preston, Some inequalities involving the Hardy-Littlewood maximal function in the theory of capacities, Proc. Sympos. Functional Analysis, Academic Press, New York, 1970.

9. P. Sjolin, Convergence almost everywhere of certain singular integrals and multiple Fourier series, Ark. Mat. 9 (1971), 65-90.

10. A. Zygmund, Trigonometrical series. Vol. II, 2nd ed., Cambridge Univ. Press, New York, 1959. MR 21 \#6498.

Department of Mathematics, Rice University, Houston, Texas 77001 